\title{
High Current, Low Voltage Current Mirrors and Applications
}

\author{
S. S. RAJPUT ${ }^{1}$ AND S. S. JAMUAR ${ }^{2}$ \\ ${ }^{I}$ Thin Film Technology group, National Physical Laboratory, Dr. K. S. Krishnan Road, New \\ Delhi 110012 India \\ email:ssrajput@csnpl.ren.nic.in \\ ${ }^{2}$ Department of Electrical Engineering, Indian Institute of Technology, Delhi, New Delhi \\ 110016, India \\ email: ssjamuar@ee.iitd.ernet.in
}

\begin{abstract}
An improved current mirror structure suitable for low voltage analog applications is proposed which has high output impedance $(\approx 5 \mathrm{M} \Omega)$, high bandwidth $(\approx 400$ $\mathrm{MHz})$, low input $(<0.4 \mathrm{~V})$ and output compliance voltages $(<0.5 \mathrm{~V})$ and high output current capability $(\approx 500 \mu \mathrm{A})$. These properties enhance the utility of this type of current mirrors. Applications of these current mirrors in analog circuits are also presented to demonstrate their superiority over their conventional counterpart. PSpice simulations confirm the suitability of the mirror for low voltage analog applications.
\end{abstract}

Key words: Analog VLSI, current mode analog signal processing, current conveyors, transconductors, current mirrors.

\section{INTRODUCTION}

Low voltage CMOS analog circuit structures are well established now. The requirement for such circuits stems from the requirements of high circuit density required to incorporate both analog and digital systems on a single chip. Hence, for mixed mode and complex circuit structures, smaller size devices are necessary. The device sizes are shrinking at a fast rate [1] and the threshold voltage of the MOSFET devices cannot be reduced below a certain minimum value. This necessitates investigation into new circuits using high 
threshold voltage MOS devices, which can operate at low voltages without sacrificing the circuit performance.

In analog circuits, a current mirror (CM) is an essential subsystem block. The overall performance of the analog circuit is dependent on the characteristics of the CM. This has generated the need for design of low voltage (LV) CM with low input and output voltage operation. Almost allhigh swing CMs, which have been reported [2-4] so far, suffers due to the requirements of high voltage at the input end. Some LVCMs have been reported, which require low input voltages [5-7] for their operation. However, these $\mathrm{CM}$ structures possess poor frequency response $(<100 \mathrm{MHz})$ along with lower input current range $(<100 \mu \mathrm{A})$. The demerits of these CMs make them unsuitable for use in low voltage high frequency analog and mixed mode signal processing applications such as current conveyors, operational floating amplifiers etc.

In this paper, we propose a LVCM circuit structure based on popular tail current enhancement technique [8,9]. This circuit has capability to operate at lower input voltages $(<0.5 \mathrm{~V})$. The proposed LVCM circuit has been obtained through suitable modifications incorporated in the circuit of $[8,9]$ by using a level shifter [5,7] at their input port. It is possible to obtain composite current outputs (i.e., positive and negative current simultaneously) by suitable modifications in output structure of LVCM. LVCMs, with composite current outputs are normally used in current mode analog signals processing circuits. The operating current range of the modified circuit extends from $1 \mu \mathrm{A}$ to $500 \mu \mathrm{A}$ with low input $(<0.4 \mathrm{~V})$ and output $(<0.5 \mathrm{~V})$ compliance voltages. The proposed LVCMs can operate up to a frequency of $400 \mathrm{MHz}$ for negative output currents. However, for the positive output current the frequency response is in the range of $200 \mathrm{MHz}$. The proposed LVCM has been used to obtain class A current conveyor (CC) and voltage to current (V-I) converters.

\section{PROPOSED LV CURRENT MIRRORS}

\section{$2.1 \quad$ CM Structure}

The proposed LVCM circuit structure is shown in Fig. 1. At the input port a PMOS transistor M9 is used to shift the level of gate bias voltage required to operate the input transistor M2 in linear (triode) region. Injection of the input current $I_{i n}$ into the drain of transistor M2 causes a voltage $\left(v_{m}\right)$, at its drain which is known as the input compliance voltage. The voltage shifter transistor M9 is designed to operate in saturation region for which an 
appropriate amount of the bias current is required to flow. The selection of this bias current depends over the compromise reached between the offset current and $v_{m}$. Input bias current is obtained from an inverted current mirror formed by M15, M16, and M17. To enhance the output impedance of the current source, a cascode structure of MOSFETs, is built using M1 and M5 at the output.

Though the cascode structure at the output port enhances the output impedance, it affects the available output signal (voltage) swing. The required gate bias for M5 is achieved through another voltage shifter PMOS (M8). A CM formed by M14, M16, and M17 provides biasing current for M8. In this configuration, the output impedance $R_{\text {out }}$ of the transistor M1 is enhanced and is given as [8]

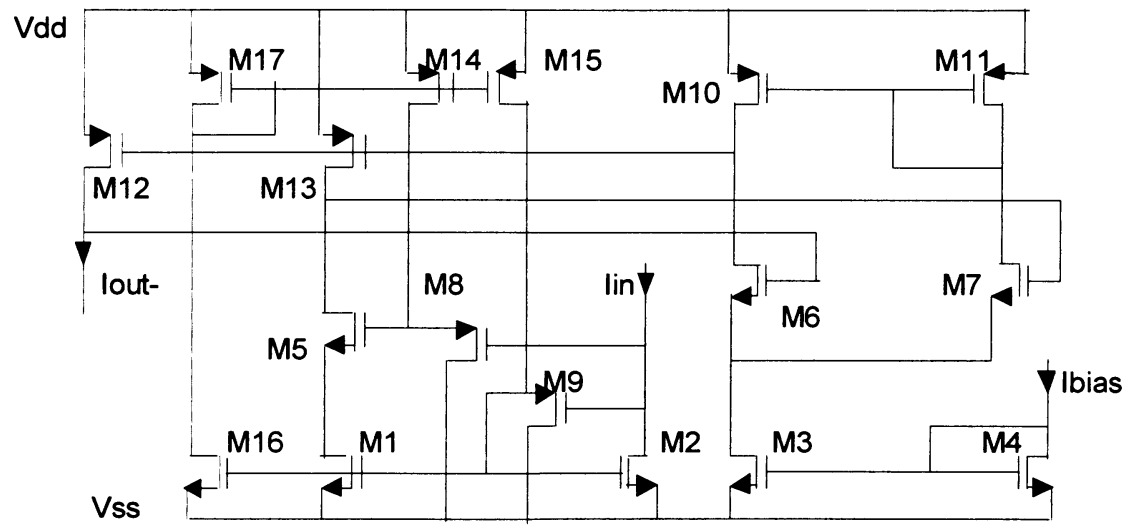

Figure 1. Proposed LV current mirror

$$
R_{\text {out }} \approx-g_{m 5} / g_{d l} g_{d s}
$$

If $g_{d \imath}$ and $g_{m ı}$ are the output conductance and trans-conductance of M2 respectively, the input impedance $R_{l n}$ and $v_{l n}$ will be given by

$$
\begin{aligned}
& R_{l n} \cup \frac{1}{g_{m l}+g_{d l}} \\
& v_{i n} \cup \Delta V_{T}+\sqrt{\Delta V_{T}^{2}+\frac{2 I_{m n}}{\beta_{n 2}}}
\end{aligned}
$$

where $\Delta V_{T}=V_{T P}-V_{T N}, V_{T P}$ and $V_{T N}$ are the threshold voltages for M9 and M2. 
Due to mismatch in the threshold voltages of the NMOS and PMOS transistors which are used in the input/output circuits and level shifter; there is an output current known as offset current $\left(I_{\text {offset }}\right)$ for zero input current. The offset current for the circuit of Fig. 1 is given by

$$
I_{\text {offset }} \cup \frac{\beta_{n 2}}{2}\left\{X+\Delta V_{T}^{2}+2 \Delta V_{T} \sqrt{X}\right\}
$$

where,

$$
X=\sqrt{\frac{2 I_{\text {blas } 1}}{\beta_{p 9}}}
$$

If $\Delta V_{T}^{2}>\left(X+2 \Delta V_{T} \sqrt{ } X\right), I_{\text {offset }}$ can not be decreased below a certain minimum level $\left(\approx \beta_{n 2}\left(\Delta V_{T}\right)^{2} / 2\right)$. The offset current is highly dependent on the mismatch $\Delta V_{T}$ between the threshold voltages of the input/output NMOS and level shifter PMOS. Smaller the mismatch, lower will be the offset current. However, if $V_{T N}$ and $V_{T P}$ can be matched, then $I_{\text {offset }}$ can be reduced quite a bit. Thus, $I_{\text {offset }}$ sets the lower limit of operating currents available from these current mirrors.

\subsection{Composite Current Mirror}

The composite current mirror (CCM) is required in many analog subsystem blocks where both positive and negative currents are needed. CCM can be derived from Fig. 1 by adding transistors M18 and M19 at the output ports as is shown in Fig. 2. M19, which is used in cascode mode, enhances the required output impedance for the positive current output. The gates of M18 and M19 are connected to the sources of transistor M9 and M8 respectively. Thus the additional transistors are not required to provide the required biases to M18 and M19. The input and output resistance remains same as that of LVCM. However, $R_{\text {out }}$ for the positive current output is given as:

$$
R_{\text {out }} \cup \frac{g_{m 19}}{g_{d 1} g_{d 19}}
$$




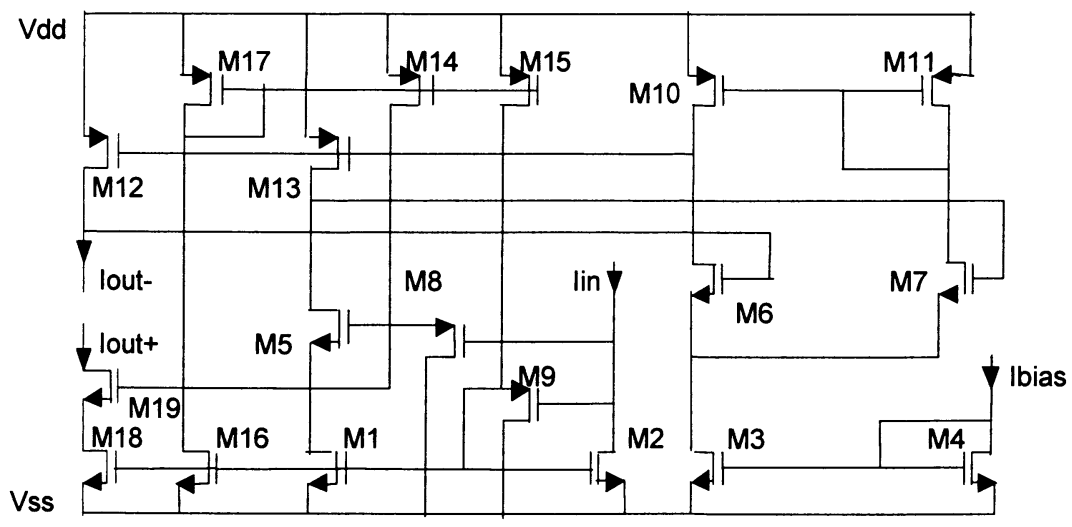

Figure 2. Proposed composite output current mirror

\section{CIRCUIT ANALYSIS}

\subsection{Modeling}

We have used hybrid $(h)$ parameters to model the CM [10] and to analyse the circuit behaviour. Small signal analysis carried out for the circuit operation at low voltages gives the following expressions for the various low frequency hybrid parameters:

$$
\begin{aligned}
& h_{11} \cup \frac{g_{d p 9}}{g_{m 2}+g_{d p 9}} \\
& h_{12} \cup 0.0 \\
& h_{21} \cup \frac{g_{m n 1}}{g_{m 2}} \\
& h_{22} \cup 0.0
\end{aligned}
$$

\subsection{Frequency Response}

Current transfer ratio from output to the input port is an important parameter for evaluation of the performance of LVCM. The frequency response characteristics in terms of output current $I_{o u t}$ to $I_{i n}$ of the proposed $\mathrm{CM}$ is given as: 


$$
\frac{I_{o u t}(s)}{I_{m}(s)} \cup \frac{1+\frac{s C_{g d p o}}{g_{m p o}}}{1+\frac{s\left(2 C_{X}+C_{g d p o}\right)}{g_{m p o}}} H(s)
$$

where $C_{g d p o}$, and $g_{m p o}$, represent the gate to drain capacitance and transconductance for M12 or M13 respectively. $C_{x}$ and $H(s)$ are representing the stray capacitance associated at the gate of M7 and the current transfer function for the input circuit formed by M1, M2, M3, M8 and M9. Generally $H(s)$ is frequency independent and taking it to be unity, equation (10) reduces to:

$$
\frac{I_{o u t}(s)}{I_{\text {in }}(s)} \cup \frac{1+\frac{s C_{\text {gdpo }}}{g_{\text {mpo }}}}{1+\frac{s\left(2 C_{X}+C_{g d p o}\right)}{g_{\text {mpo }}}}
$$

As can be seen from the equation (11), the stray capacitance $\left(C_{x}\right)$ severely degrades the high frequency response for the proposed LVCM.

\subsection{Sensitivity Analysis}

Sensitivity is one of the most important criteria used for comparing the circuit performance. We shall evaluate the sensitivity of the output current over the circuit parameters such as device dimensions and circuit structure. The sensitivity of the output current with respect to $I_{b \text { bras }}$ and $\Delta V_{T}$ is given as:

$$
\begin{gathered}
S_{I_{\text {blas }}}^{I_{\text {OUT }}} \cup \frac{\beta_{n \imath}}{2 S T_{I_{\text {blas }}}^{I_{\text {OUT }}}} \\
S_{\Delta V_{T}}^{I_{\text {OUT }}} \cup \frac{2 \beta_{n !} \Delta V_{T}}{S T_{\Delta V_{T}}^{I_{O U T}}}
\end{gathered}
$$

where the stability factors are represented as:

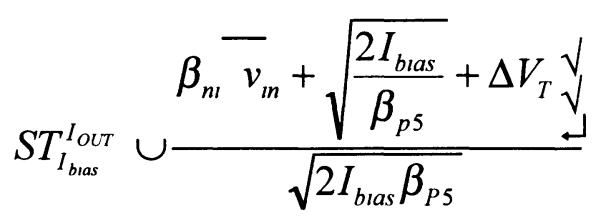


and,

$$
S T_{\Delta V_{T}}^{I_{\text {OUT }}} \cup \beta_{n t} \overline{v_{\text {in }}}+\sqrt{\frac{2 I_{\text {blas }}}{\beta_{P 5}}}+\Delta V_{T} \sqrt{ }
$$

As can be seen from the above expressions that the stability factors are quite high $\left(>10^{3}\right)$ for the proposed circuit structures. This in turn results in low sensitivity $\left(<10^{-5}\right)$ of the output current to the variations of the circuit $\left(\beta_{n}\right.$ and $\left.\beta_{p}\right)$ and device parameters $\left(\Delta V_{T}\right)$.

\subsection{Simulation Results}

The proposed LVCM circuits of Fig. 1 and Fig. 2 have been simulated using the PSPICE circuit simulator. The device parameters for $1.2 \mu \mathrm{m}$ CMOS technology [11] have been assumed. The device dimensions taken for the simulation are given in Table 1.

We are primarily interested in $R_{m}, R_{\text {out }}, v_{m}$, and the frequency response of LVCM. For the proposed structure $R_{I n}$ is equal to $700 \Omega$ (quite low value for any CMOS structure). $R_{\text {out }}$ is calculated to be $5 \mathrm{M} \Omega$ for an input (output) current of $500 \mu \mathrm{A}$, which is sufficiently higher than that of a single transistor $\mathrm{CM}$ structure. Fig. 3 shows the $I_{\text {out }}$ versus the voltage present at the drain of M12 characteristics for various values of $I_{m}$ for the proposed LVCM.

Table 1. Device dimensions

\begin{tabular}{|c|c|c|}
\hline Device & Device type & Aspect ratio (W/L) \\
\hline $\begin{array}{c}\text { M1, M2, M5, M16, } \\
\text { M18, M19 }\end{array}$ & NMOS & $240 \mu \mathrm{m} / 2.4 \mu \mathrm{m}$ \\
\hline M3, M4, M6, M7 & NMOS & $60 \mu \mathrm{m} / 2.4 \mu \mathrm{m}$ \\
\hline M8, M9, M12, M13 & PMOS & $120 \mu \mathrm{m} / 1.2 \mu \mathrm{m}$ \\
\hline M10, M11 & PMOS & $240 \mu \mathrm{m} / 2.4 \mu \mathrm{m}$ \\
\hline M14, M17 & PMOS & $240 \mu \mathrm{m} / 1.2 \mu \mathrm{m}$ \\
\hline M15 & PMOS & $1.2 \mu \mathrm{m} / 120 \mu \mathrm{m}$ \\
\hline
\end{tabular}

LVCM needs a voltage margin of less than $0.5 \mathrm{~V}$ at both ends of the power supply for an input current swing up to $500 \mu \mathrm{A}$. However, an input swing of $1.5 \mathrm{~V}$ for supply voltage of $2.0 \mathrm{~V}$ and for $I_{m}<250 \mu \mathrm{A}$ can be achieved. 


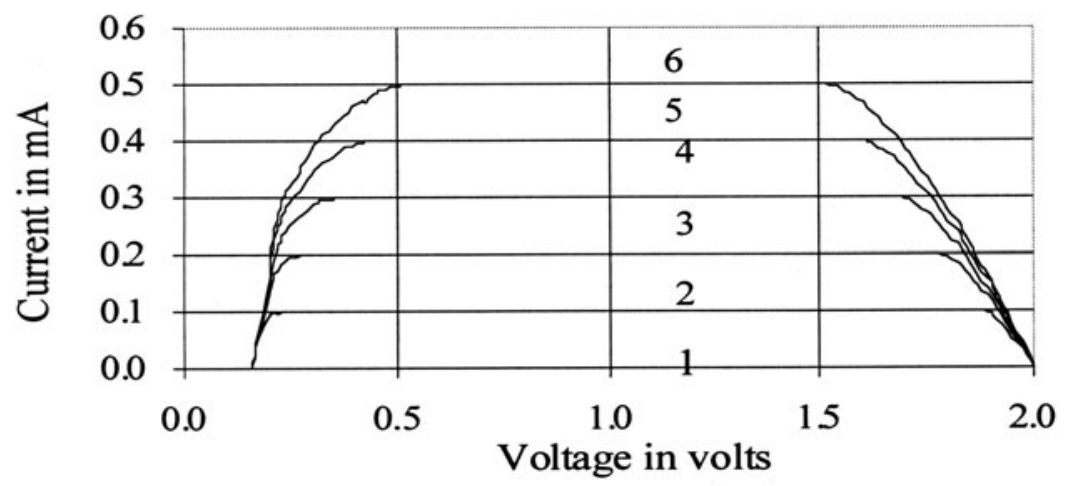

Figure 3. Output current versus the voltage present at the drain of M12 characteristics for various values of $\mathrm{I}_{\text {in }}$ (1--0.0 mA, 2--0.1 mA, 3--0.2 mA, 4--0.3 mA, 5--0.4 mA, 6--0.5 mA)

The input voltage $\left(v_{i n}\right)$ characteristics are depicted in Fig. 4 . The input voltage suddenly rises to a required voltage of $0.2 \mathrm{~V}$ as $I_{m}$ is pumped in to the CM. Then it rises with the rise in the input current and still remains fairly low $(<0.4 \mathrm{~V})$ for the entire current range from $1 \mu \mathrm{A}$ to $500 \mu \mathrm{A}$. The input compliance voltage for the conventional $\mathrm{CM}$ of [8] is also plotted in Fig. 4 for comparison. A clear gain of about $0.8 \mathrm{~V}$ in the input compliance voltage is visible.

Frequency response for the proposed LVCM and conventional CM of [8], are shown in Fig. 5. The proposed CM can operate up to $400 \mathrm{MHz}$ while the conventional $\mathrm{CM}$ without the proposed modifications has a bandwidth of $270 \mathrm{MHz}$ for same device parameters. The bandwidth enhancement due to the proposed modifications is explained in reference 12 and 13 in detail. The superiority of the proposed LVCM in terms of frequency response is evident from the figure. The peak present in the output is due to the pole shifting toward origin because of the stray capacitance $C_{x}$.

\section{LVCM APPLICATIONS}

The proposed LVCM can find use in low voltage analog signal processing circuits. We examine applications of LVCM in current conveyors and in linear trans-conductors. 


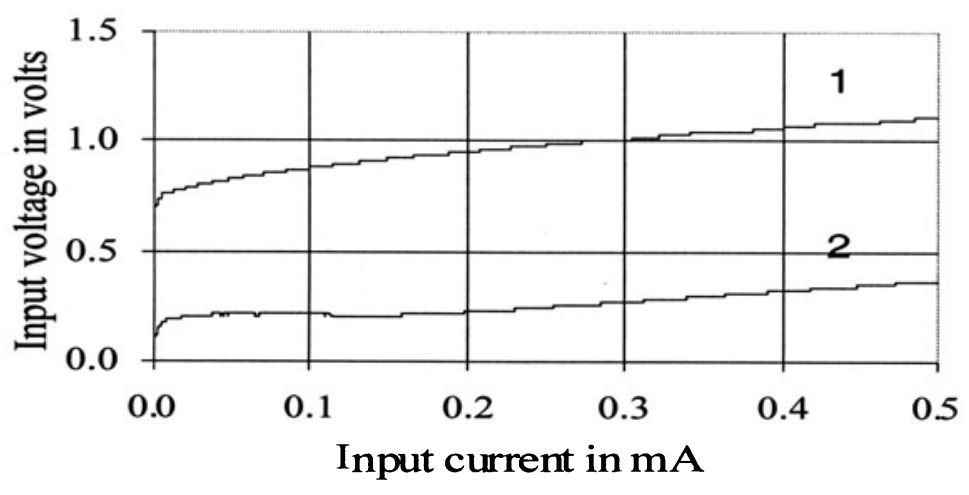

Figure 4. Input voltage characteristics (1 - Conventional mirror [8] and 2 - Proposed LV CM)

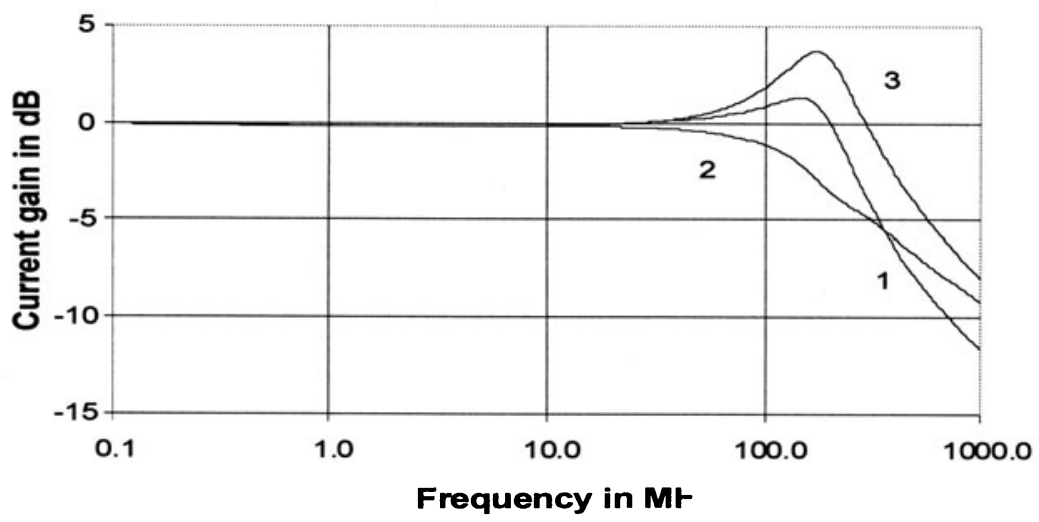

Figure 5. Simulated frequency response characteristics (1-Conventional CM [8], 2-Proposed LVCM for positive current output and 3--- for negative current output).

\subsection{Current Conveyor II}

LVCM discussed in the previous section has been used for the design of a class A CMOS current conveyor III (CCIII) of the type discussed in [14, 15]. For rail to rail operation of this type of CCIIIs, low input voltage CMs are necessary. 


\subsubsection{Circuit Implementation}

The circuit diagram of a proposed CCIII is shown in Fig. 6. The voltage transfer from port $\mathrm{Y}$ to port $\mathrm{X}$ takes place as the LVCM1 forces the equal current to flow through $M 1$ and M2. When the aspect ratio (W/L) of the transistor are kept to ensure $\beta_{n 1}=\beta_{\mathrm{p} 2}$, the drop across gate source terminals of $\mathrm{M} 1$ and M2 will be equal, which in turn transfer the voltage applied at the drain of M1 to the source terminal of M2. Current transfer from port X to port $Y$ is achieved through the action of LVCM1 and LVCM2.

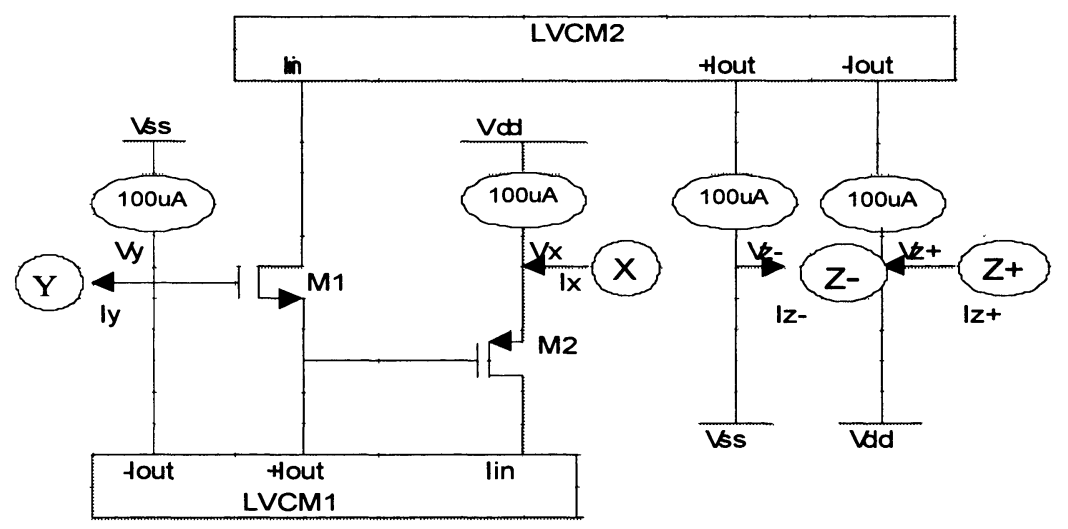

Figure 6. Proposed CCIII structure.

As evident from the figure the input signal can swing rail to rail for the positive inputs however, for negative voltages the input voltage swing is restricted to:

$$
v_{i n} \cup V_{S S}-V_{T}-V_{C M}
$$

When $V_{C M}$ is very low as in the case of proposed LVCM, the swing range increases approximately by $0.8 \mathrm{~V}$ (the difference in the input voltage of proposed and conventional $\mathrm{CMs}$ ).

\subsubsection{Simulation Results}

P-Spice simulations were carried out for CCIII structure. The aspect ratio for transistor M1 and M2 were taken to be $60 \mu \mathrm{m} / 1.2 \mu \mathrm{m}$ and $240 \mu \mathrm{m} / 1.2$ $\mu \mathrm{m}$ for NMOS and PMOS transistors respectively. The bias voltages are taken to be $\pm 1.0 \mathrm{~V}$. The output characteristics for the input current swing are 
shown in Fig. 7. The maximum input current is $\pm 100 \mu \mathrm{A}$. The voltage developed at the $\mathrm{X}$ terminal due to injected current is $100 \mathrm{mV}$.

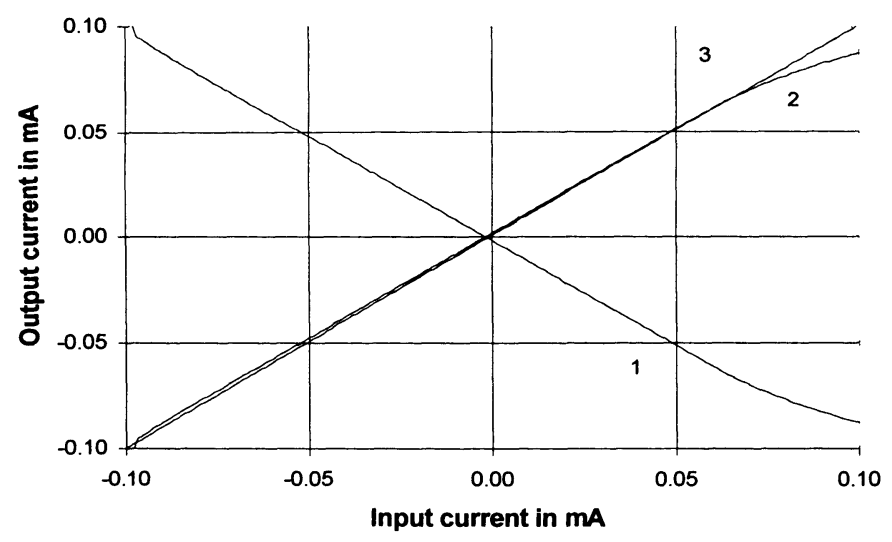

Figure 7. DC input output characteristics of the proposed CCIII

$$
\left(1---I_{Z^{+}}, 2---I_{Z_{-}}, 3---I_{Y}\right)
$$

For the frequency response characteristics of the proposed CCIII, an ac current is injected at the input port $X$, and the resultant current in the $Z+$ and $Z$ - terminals are recorded and presented. The frequency response characteristics are given in Fig. 8. The power consumption is $2.7 \mathrm{~mW}$ and the circuit has $20 \mathrm{MHz}$ bandwidth.

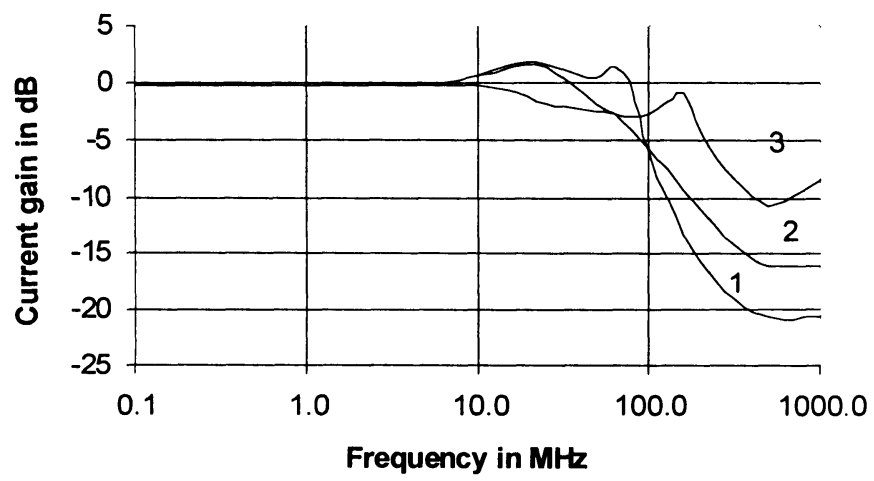

Figure 8. Frequency response of CCIII (1---Positive output current, 2--negative output current, 3---Current required at port $Y$ ). 


\subsection{Linear Voltage to Current Converter}

Voltage to current (V-I) converters are known as trans-conductors which find many uses in signal processing circuits like analog filters. High linearity, low noise, large trans-conductance and low power dissipation are the few important parameters required for a high frequency trans-conductors The proposed LVCM is used to construct a linear trans-conductor.

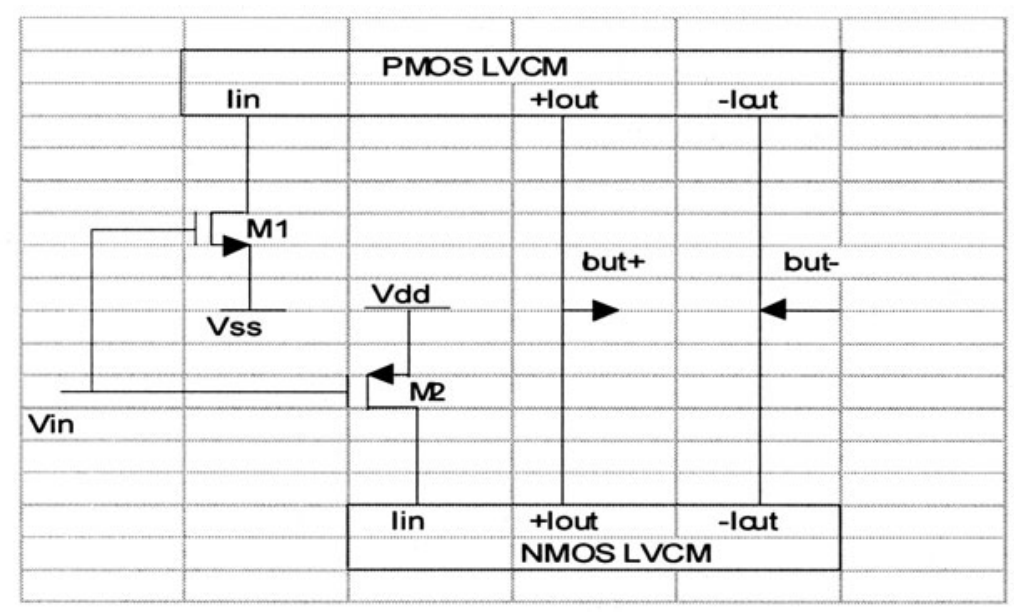

Figure 9. Proposed trans-conductor structure

\subsubsection{Circuit Implementation}

The circuit structure adapted for the realization of the trans-conductor is shown in Fig. 9. It is assumed that the transistor M1 and M2 operate in saturation, the currents flowing into the input transistors M1 and M2 are given by

$$
\begin{aligned}
& i_{D 1} \cup \frac{\beta_{n 1}}{2}\left(V_{i n 1}-V_{T N}\right)^{2} \\
& i_{D 2} \cup \frac{\beta_{p 2}}{2}\left(V_{i n 2}-V_{T P}\right)^{2}
\end{aligned}
$$

Assuming $\beta_{n 1}=\beta_{\mathrm{p} 2}=\beta, V_{m 1}=V_{I m}-V_{s s}, V_{I n 2}=V_{I n}-V_{d d}$ and $V_{d d}=-V_{s s}$, the current at the output can be given as:

$$
\begin{aligned}
& i_{C}=i_{D 1}-i_{D 2} \\
& \cup 2 \beta V_{d d}\left(V_{m}-V_{T N}\right)
\end{aligned}
$$


Examination of the above equation yields that the trans-conductor will show a linear characteristic and the trans-conductance can be varied by suitably chosen values of $\beta$ and/or supply voltages.

\subsubsection{Simulation Results}

For PSpice simulations of above trans-conductor, the aspect ratio for transistor M1 and M2 were taken to be $7.2 \mu \mathrm{m} / 2.4 \mu \mathrm{m}$ and $30.6 \mu \mathrm{m} / 2.4 \mu \mathrm{m}$ for NMOS and PMOS transistors respectively. The substrate contacts of these MOSFETs are assumed to be connected to the most positive terminal for the PMOS and the most negative terminal for the NMOS respectively and the supply voltages of $\pm 1.0 \mathrm{~V}$ are taken for all simulations.

Output current versus input voltage characteristics of the circuit is depicted in Fig. 10 in which its rail to rail input voltage swing capability is evident. The trans-conductor consumes $0.6 \mathrm{~mW}$ power.

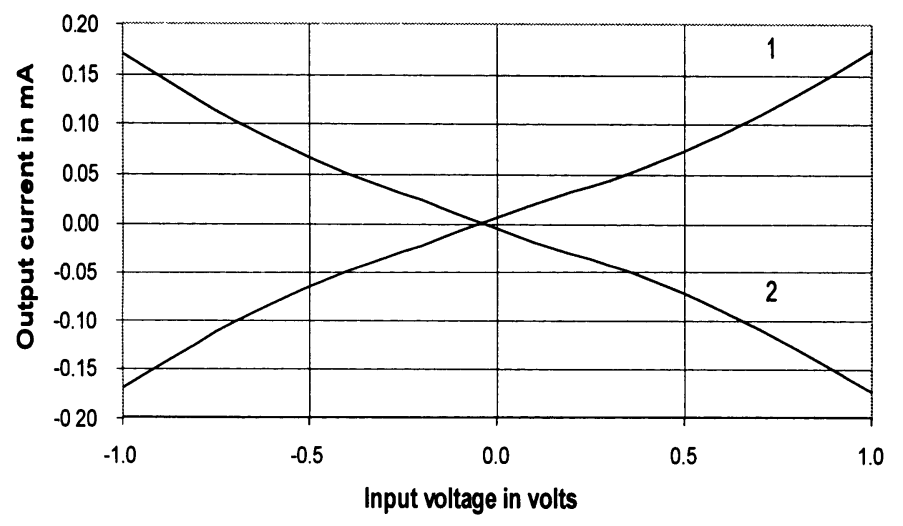

Figure 10. V-I conversion characteristics $\left(1-\mathrm{I}_{\text {out }}\right.$ and 2--- $\left.\mathrm{I}_{\text {out }}\right)$

\section{CONCLUSIONS}

We have presented a modified low voltage, current mirror structure, capable of giving high input and output voltage swings. The operating current range of the LVCM extends from $1 \mu \mathrm{A}$ to $500 \mu \mathrm{A}$ with a bandwidth as high as $200 \mathrm{MHz}$. The input voltage requirement is also quite small $(<0.4$ V). The usefulness of the proposed LVCM over its conventional part of [8] makes it an attractive candidate for low voltage analog design.

The superiority of these LVCMs when used in the current conveyor structures and linear trans-conductor structures is also significant. The resultant CCIII and trans-conductor structures consume very low power and 
can operate at sufficiently low voltages present at the input port. Thus the proposed LVCM can be used for low voltage, analog circuit applications.

\section{ACKNOWLEDGEMENT}

One of the author (S. S. Rajput) expresses his gratitude to Dr. R. Bhattacharyya, Head, Thin Film Technology Group, NPL and to Prof. A. K. Raychodhury, Director NPL New Delhi, for their constant encouragement, and keen interest in the work.

\section{REFERENCES}

[1] Y. Taur, D. A. Buchanan, W. Chen, D. J. Frank, K. E. Ismail, S. Lo, G. A. Sai-halisz, R. G. Vishvanathan, H. C. Wann, S. J. Wind, and H. Wang, "CMOS scaling into the nano-meter regime", Proc. IEEE, April 1997, 486-504.

[2] J. Mulder, A. C. Woerd, W. A. Serdian, and A. H. M Roermund, "High swing cascode MOS current mirror", Electron Lett. , $4^{\text {th }}$ July, 1996, 32, 1251-1252

[3] E. Sackinger, and W. Guggenbuhl, "A high swing, high impedance MOS cascode current mirror", IEEE J. Solid State Circuits, Feb. 1990, 25, 289-298

[4] A. Zeki, and H. Kuntman, "Accurate and high output impedance current mirrors suitable for CMOS current output stages", Electron Lett. , $5^{\text {th }}$ June 1997, 33, 1042-1043

[5] V. I. Proddanov, and M. M. Green, "CMOS current mirrors with reduced input and output voltage requirements", Electron Lett. , $18^{\text {th }}$ Jan. 1996, 32, 104-105

[6] P. Heim, and M. A. Jabri, "MOS cascode-current mirror biasing circuit operating at any current level with minimal output saturation voltage", Electron Lett, 1995, 31, 690-691

[7] B. J. Blalock, P. E. Allen, and A. R. Gariel, "Designing 1-V Op Amps using standard digital CMOS technology", IEEE Trans. Circuits and Systems-I, July 1998, 45, 769-780.

[8] F. You, S. H. K. Embabi, J. F. Duque-Carrilo, and E. Sanchez-Sinencio, "An improved tail current source for low voltage applications", IEEE J. Solid State Circuits, Aug. 1997, 37(8), 1173-1179

[9] E. Seevinck, M Plessis, T. Joubert, and A. E. Theron, "Active bootstrapped gain enhancement technique for low voltage circuits", IEEE Trans. Circuits Systems-II, Sept. 1998, 45(9), 1250-1254

[10] Jaeger, R. C., Microelectronics Circuit Design, Mc-Graw Hill, New York, 1997

[11] Silval-Martinez, J., Steyaert, M., Sansen, W., High performance CMOS continuous time filters, Kluwer Academic Publishers, Boston, 1993

[12] T. Voo, and C. Toumazau, 'High speed current mirror resistive compensation technique', Electron Lett., 1995, 31 , no. 4, pp. 248-250

[13] T. Voo, and C. Toumazau, "Precision temperature stabilized tunable CMOS current mirror for filter applications", Electron Lett., 1996, 32 , no. 2, pp. 105-106

[14] H. W. Cha, and K. Watanabe, "Wide band CMOS current conveyor", Electron Lett., vol. 32, no. 14, pp. $1245-1246,4^{\text {th }}$ July 1996

[15] S. S. Rajput, and S. S. Jamuar, "A new wide bandwidth current controlled CMOS current conveyor III implementation," reported to Electronics letters. 